\title{
Análisis factorial confirmatorio de la Escala de Involucramiento Escolar para Adolescentes (EIE-A)
}

Confirmatory factor analysis of Adolescent's School Engagement Scale (ASES)

\section{Volumen 20, Número 1 \\ Enero - Abril \\ pp. 1-18}

\author{
María Enriqueta Sánchez Hernández \\ Patricia Andrade Palos
}

\section{Citar este documento según modelo APA}

Sánchez Hernández, María Enriqueta y Andrade Palos, Patricia. (2020). Análisis factorial confirmatorio de la Escala de Involucramiento Escolar para Adolescentes (EIE-A). Revista Actualidades Investigativas en Educación, 20(1), 1-18. Doi. 10.15517/aie.v20i1.40020 


\title{
Análisis factorial confirmatorio de la Escala de Involucramiento Escolar para Adolescentes (EIE-A) \\ Confirmatory factor analysis of Adolescent's School Engagement Scale (ASES)
}

\author{
María Enriqueta Sánchez Hernández ${ }^{1}$ \\ Patricia Andrade Palos ${ }^{2}$
}

\begin{abstract}
Resumen: El involucramiento escolar comprende las conductas, creencias y emociones de la población estudiantil hacia su actividad escolar. Es un constructo que se ha investigado ampliamente en el alumnado de educación superior y con menor frecuencia en estudiantes de educación básica. Las personas adolescentes con mayor involucramiento escolar tienen mejor desempeño y se involucran menos en conductas de riesgo, por lo que resulta importante investigar más acerca de este tema. El objetivo del presente artículo es presentar las propiedades psicométricas de un instrumento para evaluar el involucramiento escolar en el estudiantado de secundaria. Esta investigación cuantitativa se realizó con población estudiantil de México y los datos se obtuvieron entre 2018 y 2019. La muestra no probabilística estuvo constituida por 503 estudiantes de secundarias públicas de la Ciudad de Xalapa, Veracruz; 57.5\% fueron mujeres y 42.5\% hombres, con edades entre 11 y 15 años ( $M=13.12$, D.E. 0.92). La versión final de la escala está compuesta de diez reactivos con siete opciones de respuesta que evalúan tres dimensiones: conductual, emocional y cognitiva. El análisis factorial confirmatorio mostró índices de ajuste adecuados $C F I=0.98, T L I=0.98, S R M R=0.03, R M S E A=0.05$, y los coeficientes de confiabilidad interna fueron $\alpha=0.88$ y $\lambda_{2}=0.88$. Los resultados indican que la EIE-A es un instrumento adecuado para evaluar el involucramiento escolar en personas adolescentes tempranas.
\end{abstract}

Palabras clave: Involucramiento escolar, adolescencia, medición, rendimiento escolar.

\begin{abstract}
School involvement includes the behaviors, beliefs and emotions of student population have towards their school activity, it is a construct that has been widely studied in college students but infrequently in middle school students. Adolescents that are more engaged in school activities demonstrate better performance in class and are less prone to get involved in risk behaviors, which is why a more in-depth study is needed. The objective of this article is to present the psychometric properties of an instrument to evaluate students' school engagement at secondary school level. This quantitative research was carried out with students from Mexico and the data were obtained between 2018 and 2019. The non-probabilistic sample consisted of 503 public high school students from the City of Xalapa, Veracruz; 57.5\% were women and $42.5 \%$ men, aged between 11 and 15 years $(M=13.12$, D.E. 0.92). The final version of the scale contains ten items, each with seven possible responses which evaluates 3 dimensions: behavioral, emotional and cognitive. The confirmatory factor analysis showed adequate goodness of fit indexes CFI $=0.98, T L I=0.98, S R M R=0.03, R M S E A=0.05$ and the coefficients of reliability were $\alpha=0.88 y$ $\lambda_{2}=0.88$. The results indicate that the ASES is a suitable instrument to evaluate early adolescence school involvement.
\end{abstract}

Key words: school engagement, adolescence, measurement, school performance.

\footnotetext{
${ }^{1}$ Candidata a doctorado de la Universidad Nacional Autónoma de México, en la Facultad de Psicología, México. Maestría por la Universidad Veracruzana, México. Dirección electrónica: meshmt@hotmail.com ORCID : https://orcid.org/0000-0003-1043-4905

${ }^{2}$ Profesor titular C T.C. de la Universidad Nacional Autónoma de México, en la Facultad de Psicología, México. Doctora en psicología social por la UNAM. Dirección electrónica: p.andradepalos@gmail.com ORCID : https://orcid.org/0000-0003-0072-1169
}

Artículo recibido: 8 de julio, 2019

Enviado a corrección: 18 de setiembre, 2019

Aprobado: 14 de octubre, 2019 


\section{Introducción}

Existen diferentes formas de referirse al involucramiento escolar (Daura, 2015), Appleton, Christenson y Furlong (2008) sintetizaron la información de varios estudios y encontraron ocho denominaciones distintas y 19 definiciones diferentes. Algunos ejemplos de la variedad de términos son: involucramiento escolar (Peña et al., 2017), implicación escolar (Ramos-Díaz, Rodríguez-Fernández, Ros y Antonio-Aguirre, 2016), involucración académica (Daura, 2015), compromiso con el aprendizaje (Benson, 2007), compromiso académico (Libbey, 2004; Kim y Suárez-Orozco, 2015), compromiso escolar (Fredricks, Blumenfeld y Paris, 2004; Glanville y Wildhagen, 2007; Schaufeli, Salanova, González-Romá y Bakker, 2002; Wang y Eccles, 2012) y compromiso estudiantil en la escuela (Veiga y Robu, 2014). Ante esta situación, Appleton et al. (2008) insisten en que es importante contar con mayor evidencia que ayude a clarificar el constructo de manera empírico-teórica.

Daura (2015) y Finn (1993) señalan que el compromiso y el involucramiento escolar son sinónimos, sin embargo el compromiso escolar (school engagement o student engagement en los estudios de habla inglesa) se emplea más que involucramiento escolar para referirse al mismo tema (Fredricks y McColskey, 2012). En este estudio se decidió usar el concepto involucramiento porque la palabra compromiso, en español, hace referencia a una obligación (Real Academia Española, 2019), lo cual no corresponde con la naturaleza del constructo.

La investigación psicoeducativa se ha centrado en un modelo de riesgo (FernándezZabala, Goñi, Camino y Zulaika, 2016) que estudia temas como el fracaso escolar (Tarabini, 2015), la victimización escolar (Buelga, Cava y Musitu, 2012), la deserción escolar (Peña, Soto y Calderón, 2016), entre otros. En oposición a esta perspectiva, Fernández-Zabala et al. (2016) señalan que: "la psicología positiva se interesa en la adaptación, considerando el compromiso estudiantil como factor importante de esta" (p. 48). Congruente con esta postura alternativa, en la actualidad son numerosas las investigaciones que exploran factores positivos asociados al involucramiento escolar, por ejemplo, existen estudios que investigan si el compromiso escolar puede considerarse una medida de ajuste psicosocial (RodríguezFernández et al., 2016).

El involucramiento escolar es un constructo importante de analizar, ya que se asocia con resultados positivos, por ejemplo, existe evidencia empírica que muestra que el compromiso escolar es un predictor de alto rendimiento académico (Kim y Suárez-Orozco, 2015; Lam et al., 2014; Pan y Zaff, 2017; Parra, 2010; Peña, Cañoto y Angelucci, 2017; 
Wang y Holcombe, 2010), más satisfacción con la escuela (Gutiérrez, Tomás, Romero y Barrica, 2017), éxito académico, más satisfacción con la vida, menos síntomas depresivos (Upadyaya y Salmela-Aro, 2013) y menor nivel de burnout, referido como cansancio emocional crónico, pérdida de realización personal y despersonalización (Parra y Pérez, 2010). Otros estudios señalan que el estudiantado con mayor puntaje en involucramiento escolar tiene un desarrollo favorable y reduce sus conductas de riesgo (Dolzan, Sartori, Charkhabi y De Paola, 2015), y quienes tienen una menor puntuación en compromiso escolar comportamental y emocional muestran aumento en puntajes de delincuencia y consumo de sustancias (Wang y Fredricks, 2014).

Si bien existen algunos instrumentos en español que contemplan las tres dimensiones más utilizadas para medir el constructo, es decir, los aspectos cognitivos, emocionales y conductuales (Estell y Perdue, 2013), no se consideró pertinente retomarlos ya que miden el aspecto cognitivo con el autoreporte de conductas relacionadas con el esfuerzo para aprender, lo cual debería evaluarse a través de la ejecución de tareas (Embretson y Gorin, 2001). En esta investigación la dimensión cognitiva se evaluó con base en las creencias que el alumnado tiene hacia su actividad escolar.

Este artículo presenta el análisis de las propiedades psicométricas de un instrumento que mide involucramiento escolar, definido como el conjunto de acciones (dimensión conductual), creencias (dimensión cognitiva) y emociones (dimensión emocional) que tiene el estudiantado ante su actividad escolar. Se trata de una investigación cuantitativa que se realizó en 2018-2019 con población estudiantil de la ciudad de Xalapa, Veracruz en México.

\section{Referente teórico}

Existen tres distintos modelos teóricos que se han utilizado para definir el involucramiento escolar: teoría constructivista, teoría sociocognitiva y teoría sociocrítica (Daura, 2015). Lo anterior ha dado lugar a múltiples definiciones, a continuación se presentan algunas de estas respetando la denominación empleada por cada persona autora, en las que se pueden apreciar diferencias conceptuales.

Para Peña et al. (2017) el involucramiento académico se refiere a la inversión de tiempo, esfuerzo, recursos físicos y psicológicos por parte del estudiantado y la institución, para optimizar la experiencia académica y mejorar el resultado de aprendizaje, el desarrollo del estudiantado y la reputación de la institución. Esta concepción de involucramiento escolar 
implica a la población escolar y a la escuela, a diferencia de las siguientes que se enfocan especialmente en el estudiantado.

Desde el enfoque del Desarrollo Positivo del Adolescente (PYD por sus siglas en inglés) y en el marco del modelo de fortalezas del desarrollo (Benson, 2007), se hace referencia a compromiso con el aprendizaje como la combinación de creencias, habilidades y valores personales del adolescente hacia el aprendizaje; se evalúa a través de cinco fortalezas internas o recursos personales: 1) motivación por los logros académicos (motivación para hacer bien las cosas en la escuela), 2) compromiso escolar (participar activamente en los procesos de aprendizaje), 3) tareas (destinar una o más horas para realizar las tareas), 4) lazos con la escuela (preocuparse por la escuela) y 5) placer por la lectura (leer por placer tres o más horas a la semana).

Parra (2010) y Schaufeli et al. (2002) mencionan que el compromiso escolar es un estado mental positivo relacionado con el trabajo académico, el cual se compone de tres factores: 1) vigor (altos niveles de energía y resistencia mental para desempeñar una labor específica), 2) dedicación (alto nivel de implicación laboral, sentido de significación, entusiasmo, inspiración, orgullo y reto) y 3) absorción (alto estado de concentración e inmersión en la tarea). En esta definición se puede observar mayor énfasis en aspectos cognitivos, a diferencia de las siguientes definiciones que consideran de considerar factores conductuales e interpersonales.

Glanville y Wildhagen (2007) señalan que el compromiso escolar es la medida en que el alumnado participa en los programas académicos y actividades no académicas de la escuela, se sienten conectados con la escuela y valoran los objetivos de la educación. Esta definición es parecida a la definición de Wang y Eccles (2012), quienes indican que el compromiso escolar implica participación escolar, sentido de pertenencia a la escuela y aprendizaje autorregulado.

Una concepción más es la de Fredricks et al. (2004), quienes señalan que el compromiso escolar tiene tres componentes que varían en intensidad y duración: 1) compromiso comportamental (asistencia escolar y participación en actividades académicas y sociales o extracurriculares), 2) compromiso emocional (reacciones positivas y negativas hacia el personal docente, el alumnado y la escuela, los cuales crean vínculos con la institución e influyen en la voluntad de trabajar) y 3) compromiso cognitivo (consideración y voluntad de esforzarse lo necesario para comprender las ideas complejas y dominar las habilidades difíciles). Estell y Perdue (2013) sostienen que la perspectiva de tres 
dimensiones: conductual, emocional y cognitiva es la más utilizada. Por su parte, Daura (2015) señala que es la más exhaustiva, por considerar aspectos intrapsíquicos y factores contextuales; de ahí que muchas investigaciones la retoman, por ejemplo el estudio de Lam et al. (2014) en el que participaron estudiantes de 12 países.

Las definiciones mencionadas ponen de manifiesto que delimitar el involucramiento escolar es complejo, pues hay quienes lo consideran como un proceso (Christenson, Reschly y Wylie, 2012; Janosz, 2012) y otros como un resultado (Appleton et al., 2008; Janosz, 2012). Si bien, hay acuerdo en que es un constructo multidimensional (Christenson et al., 2012; Fredricks et al., 2004; Fredricks y McColskey, 2012; Hernández, 2015; Janosz, 2012, Owen et al., 2016) no existe consenso respecto al número y tipo de dimensiones (Fredricks y McColskey, 2012; Hernández, 2015; Skinner, Furrer, Marchand y Kinderman, 2008).

Así como existen varias definiciones del involucramiento escolar, también existe una variedad de instrumentos que lo evalúan en muestras de diferentes edades y países. Entre las escalas que se han utilizado con estudiantes de secundaria de 11 a 15 años de edad, población de interés en este estudio, se encuentran las siguientes.

En 2016, Veiga desarrolló la escala Students' Engagement in School Four-dimensional Scale (SES- 4DS) para medir el compromiso del estudiantado en la escuela, con base en la definición de Fredicks et al. (2004). La muestra fue de 685 adolescentes de Portugal entre 11 y 16 años de edad, que cursaban $6^{\circ}, 7^{\circ}, 9^{\circ}$ y $10^{\circ}$ grado (equivalente a primero y segundo grado de secundaria y primero y segundo grado de bachillerato en México), el 56\% fueron mujeres y el $44 \%$ hombres. La SES-4DS tiene 20 reactivos, 15 se refieren a compromiso escolar (5 a la dimensión emocional, 5 a la cognitiva y 5 a la conductual) y cinco reactivos evalúan el constructo de agencia. Los índices de ajuste fueron adecuados para el modelo ( $X 2$ $=792.73, d f=164, \mathrm{p}<.001, S R M R=0.07, C F I=0.87, R M S E A=0.075$ Cl 90\% 0.07-0.08). El alfa de Cronbach de la escala total fue 0.82 y las alfas de las subescalas se encontraron entre 0.70 y 0.85 . Como evidencia de validez de criterio se señaló que estudiantes con mayor compromiso tenían menos retenciones y que el compromiso tuvo correlaciones positivas con las calificaciones en las asignaturas de matemáticas y portugués.

Hazel et al. (2013) propusieron un modelo para medir compromiso escolar estudiantil con base en el modelo de compromiso escolar de Fredricks et al. (2004) y el modelo de compromiso estudiantil de Christenson et al. (2012). Asimismo, llevaron a cabo un estudio con 396 estudiantes de Estados Unidos de América, de 13 y 14 años de edad, que cursaban $8^{\circ}$ grado (equivalente a tercer grado de secundaria en México) para buscar evidencias de 
validez del Instrument Student School Engagement Measure (SSEM). La versión final del instrumento contó con 22 reactivos que evalúan: a) aspiraciones, b) pertenencia y c) productividad. Los índices de ajuste fueron adecuados para el modelo de tres dimensiones $(C F I=0.92, G F I=0.88, S R M R=0.05, R M S E A=0.07 C l 90 \% 0.06-0.07)$, el rango de la confiabilidad de las subescalas fue de 0.83 a 0.92 , y se contó con evidencia de validez de criterio dado que se encontró correlación positiva y significativa con el rendimiento académico. En la aplicación del SSEM se presentaron los reactivos simultáneamente en español e inglés, pero no se evaluó el lenguaje que se usó para responder, por lo tanto se desconoce si el aspecto cultural asociado a la diversidad lingüística tuvo impacto en las evidencias de confiabilidad y validez del instrumento.

Skinner, Kindermann y Furrer (2009) diseñaron el Engagement versus Disaffection with learning (EvsD) con base en las teorías de motivación y lo aplicaron a 1,018 estudiantes entre nueve y 12 años de edad de Estados Unidos de América. Este instrumento cuenta con 24 reactivos y evalúa compromiso conductual (esfuerzo, atención y persistencia en iniciar y participar en actividades de aprendizaje), compromiso emocional (las emociones favorecen la motivación durante las actividades de aprendizaje), desafío conductual (falta de esfuerzo y abandono de las actividades de aprendizaje) y desafío emocional (las emociones favorecen el abandono de las actividades de aprendizaje). El análisis factorial confirmatorio mostró índices de ajuste adecuados (compromiso conductual $C F I=1.00, T L I=1.00, R M S E A=0.05$ $C l=0.01,0.09$; desafección conductual $C F I=1.00, T L I=0.99, R M S E A=0.08 \mathrm{Cl} 90 \% 0.05-$ 0.12; compromiso emocional $C F=1.00, T L I=0.99, R M S E A=0.08 C I 90 \% 0.06-0.10$; desafección emocional $C F I=1.00, T L I=0.99, R M S E A=0.05 C l 90 \% 0.04-0.07)$ y la confiabilidad reportada fue de 0.61 a 0.85 para las cuatro subescalas. Además, se tuvo evidencia de validez al correlacionar los puntajes de compromiso con factores individuales (dominio académico, optimismo y pesimismo académico, autonomía, orientación al logro, reacción a retos académicos y sentido de pertenencia) y contextuales (apoyo motivacional de la familia y del personal docente).

El Motivation and Engagement Scale (MES) evalúa la motivación y compromiso como factores adaptativos que influyen en la finalización de tareas y participación en clase (Martin, 2009). La MES cuenta con 11 subescalas de motivación y compromiso que se agrupan en dos factores cognitivos y dos conductuales: comportamiento adaptativo (planificación, gestión de tareas, persistencia), cognición adaptativa (autoeficacia, valoración, orientación de dominio), comportamiento inadaptado (discapacidad y desvinculación) e impedimento 
cognitivo (ansiedad, evitación de fallos, control incierto). El instrumento cuenta con 44 reactivos y se aplicó a 624 estudiantes de Australia de educación básica de nueve a 11 años, 21,579 estudiantes de 12 a 18 años y 420 estudiantes de educación superior. Las alfas de Cronbach para las subescalas de la versión de secundaria fueron de 0.61 a 0.81, y en el análisis factorial confirmatorio se encontró que el modelo de 11 dimensiones tenía índices de ajuste adecuados $(C F I=0.98, R M S E A=0.04)$. El MES mostró invariabilidad de su estructura factorial en diferentes niveles educativos desde la primaria hasta la universidad.

El Student Engagement Instrument (SEI) (Appleton, Christenson, Kim y Reschly, 2006) evalúa el compromiso cognitivo y el compromiso psicológico. El SEI se aplicó a una muestra de 1,931 estudiantes entre 15 y 16 años de edad de Estados Unidos de América del 9º grado (equivalente a 1er. grado de bachillerato en México,) de escuelas urbanas, étnicamente diversas y de bajos ingresos. El instrumento tiene 35 reactivos y en el análisis factorial confirmatorio mostró índices de ajuste adecuados $(C F I=0.96, T L I=0.96, R M S E A=0.06 \mathrm{Cl}$ $90 \%$ 0.01, 0.09) para seis factores, tres relacionados con el compromiso psicológico: relaciones entre estudiantes y personal docente, apoyo entre pares para aprender y apoyo familiar para el aprendizaje; y tres relacionados al compromiso cognitivo: control y relevancia del trabajo escolar, aspiraciones y objetivos futuros, y motivación extrínseca. Las alfas de Cronbach para cada subescala fueron entre 0.76 y 0.88 , y la validez convergente y discriminante se obtuvo al encontrar correlaciones positivas con desempeño en lectura y matemáticas y correlación negativa con número de suspensiones.

Tomás, Gutiérrez, Sancho, Chireac y Romero (2016) analizaron las evidencias de validez y confiabilidad de la versión en español del SES-4DS, en una muestra de 1138 estudiantes con media de edad de 15.5 años, residentes de Santo Domingo, República Dominicana. Confirmaron la estructura factorial del instrumento y obtuvieron índices de ajuste adecuados $(C F I=0.87, S R M R=0.07, R M S E A=0.075 \mathrm{Cl} 90 \%$ 0.07-0.08) al eliminar dos reactivos de la versión original. Como indicadores de confiabilidad, se reportó que las alfas de las subescalas se encontraban entre 0.62 y 0.76 , y los $C R I$ (Composite Reliability Index) entre 0.65 y 0.84 . Obtuvieron la validez de criterio a través de correlaciones positivas y significativas con orientación al desempeño, apoyo a la autonomía y satisfacción con la escuela.

Lara et al. (2018) elaboraron un cuestionario de 29 reactivos para medir las dimensiones conductual, afectiva y cognitiva del compromiso escolar. Las propiedades psicométricas del cuestionario se analizaron en una muestra de 454 estudiantes de Chile con 
media de edad de 13.51 años y niveles de vulnerabilidad estudiantil alta o extrema. El análisis factorial confirmatorio del modelo de tres factores tuvo índices de ajuste adecuados $(C F I=0.95, T L I: 0.93, R M S E A=0.05)$ y las subescalas mostraron alfas ordinales entre 0.83 y 0.87. Además, se contó con evidencia de validez externa porque los puntajes de compromiso escolar y rendimiento académico indicaron correlaciones positivas y significativas.

De los instrumentos descritos, se puede señalar que: a) la mayoría contempla aspectos conductuales, afectivos y cognitivos; sin embargo, algunos incluyen otras dimensiones como motivación, agencia personal y apoyo familiar; y b) aunque en todos los estudios se reportan índices de ajuste adecuados, hay diversidad en el número de reactivos y dimensiones que evalúan. En la literatura no se encontró un instrumento que evaluara el involucramiento escolar en población estudiantil de secundaria de México, por lo tanto el objetivo de esta investigación fue elaborar una escala dirigida a esta población. Se buscó que la escala midiera las tres dimensiones del involucramiento escolar (conductual, emocional y cognitiva) que más se han utilizado para evaluar este constructo (Estell y Perdue, 2013), con la variación de que la dimensión cognitiva se refiere a las creencias que el estudiantado tiene acerca de su actividad escolar.

\section{Metodología}

\subsection{Enfoque}

Esta investigación es de tipo cuantitativa, se realizó en 2018-2019 con población estudiantil de la ciudad de Xalapa, Veracruz en México. Se reporta el análisis factorial confirmatorio de una escala para medir involucramiento escolar en población adolescente de 11 a 15 años.

\subsection{Unidades de análisis}

La muestra no probabilística estuvo constituida por 503 estudiantes de cuatro escuelas secundarias públicas de la Ciudad de Xalapa, Veracruz. Participaron estudiantes de los tres grados escolares (37\% primero, 32.4\% segundo, 30.6\% tercero) con edades entre los $11 \mathrm{y}$ 15 años $(M=13.03, D E=0.91)$, el $57.5 \%$ fueron mujeres y el $42.5 \%$ hombres.

Se contó con la autorización de la dirección de cuatro escuelas secundarias, con el consentimiento informado de las familias y el acuerdo del estudiantado para resolver el 
instrumento. La escala fue anónima y se garantizó la confidencialidad de la carta de consentimiento informado.

\subsection{Técnicas de recolección}

Se utilizó una escala que consta de diez reactivos tipo Likert con siete opciones de respuesta (siempre, casi siempre, muchas veces, ni muchas ni pocas veces, pocas veces, casi nunca, nunca), que corresponden a tres factores de involucramiento escolar: conductual (cuatro reactivos), emocional (tres reactivos) y cognitivo (tres reactivos).

Cabe mencionar que la escala de diez reactivos se obtuvo como resultado de dos estudios previos donde se analizó la discriminación de los reactivos. En un primer momento y con base en la definición de las dimensiones del involucramiento escolar, se redactaron 67 reactivos que corresponden a las tres dimensiones del constructo: involucramiento conductual (23 reactivos), involucramiento cognitivo (20 reactivos) e involucramiento emocional (24 reactivos). A partir de la evaluación de tres jueces expertos en el tema se eliminaron 27 reactivos. Posteriormente se realizó un estudio piloto con el instrumento de 40 reactivos, el cual se aplicó a una muestra de 439 estudiantes de escuelas secundarias públicas. Una vez recabada la información se eliminaron aquellos reactivos que cumplían con alguno de los siguientes criterios: a) tener frecuencias igual o mayores al $50 \%$ en una de las opciones de respuesta, b) presentar valores de sesgo y curtosis igual o mayor a 2 y c) presentar bajas correlaciones con el puntaje total (menor a .40). Con base en estos criterios se eliminaron 24 reactivos.

Con la escala de 16 reactivos se realizó otro estudio piloto que se aplicó a una muestra de 500 estudiantes de secundarias públicas de la ciudad de Xalapa, Veracruz. Nuevamente se analizaron los reactivos con los criterios anteriores y se eliminaron seis reactivos, lo que permitió contar con la escala de diez reactivos, a la que se denominó Escala de Involucramiento Escolar para Adolescentes (EIE-A).

\subsection{Procesamiento de análisis}

Antes de realizar el Análisis Factorial Confirmatorio $(A F C)$ se calculó el valor de adecuación muestral de Kaiser-Meyer-Olkin (KMO) que fue de 0.89 , la significancia de la prueba de esfericidad de Bartlett que fue $p<0.001$, y la prueba de Mardia que mostró que los datos no tenían normalidad multivariada. 
El Análisis Factorial Confirmatorio se llevó a cabo con el programa R versión 3.5.1. Se utilizó el método de estimación de mínimos cuadrados no ponderados, el cual se recomienda para variables observadas de tipo ordinal y no requiere una distribución normal de los datos. Para determinar el ajuste del modelo propuesto, se analizaron los siguientes índices (Hu y Bentler, 1999): prueba de bondad de ajuste $X^{2}$; al tratarse de un parámetro sensible al tamaño de la muestra se consideró el cociente $X^{2} / g l(C M I N / D F)$ cuyo valor menor a 3 indica un ajuste adecuado; los índices: CFI (Comparative Fit Index), TLI (Tucker-Lewis Index), que con valores mayores a .90 se interpretan como propios de un ajuste adecuado; y los de error: RMR (Root Mean Square Residual), RMSEA (Root Mean Square of Aproximation), que muestran un ajuste adecuado si su valor es menor a .05 en el primer caso, y menor a .08, en el segundo.

Como los reactivos de la escala se encontraban en el nivel ordinal de medición, se calculó la consistencia interna a partir de una matriz de correlaciones policóricas y se obtuvieron dos indicadores de consistencia: el alfa de Cronbach y el lambda 2, que es un índice de cota más estricto.

\section{Resultados}

La Escala de Involucramiento Escolar para Adolescentes (EIE-A) que sigue un modelo de involucramiento escolar de tres dimensiones (conductual, emocional y cognitivo) obtuvo índices de ajuste adecuados: $X^{2}(g l)=77.38$ (32), $C M I N / D F=2.42, C F I=0.98, T L I=0.98$, $S R M R=0.03$ y $R M S E A=0.05(I C=0.03-0.06)$. Los coeficientes de consistencia interna de la EIE-A fueron $\alpha=0.88$ y $\lambda_{2}=0.88$. Para cada una de las dimensiones de la escala los coeficientes de consistencia interna fueron: dimensión conductual $\lambda_{2}=0.86$, emocional $\lambda_{2}=$ 0.83 y para la cognitiva $\lambda_{2}=0.74$.

En la Figura 1 se muestran las correlaciones entre los factores y los coeficientes factoriales estandarizados de los reactivos, los cuales se encontraron entre 0.63 y 0.84 . Como se puede apreciar, las correlaciones entre las tres dimensiones de involucramiento escolar fueron positivas y moderadas, tal como se esperaba por tratarse de un constructo multidimensional. La correlación más alta se encontró entre las dimensiones emocional y cognitiva $\left(r_{S}=0.74, p<.01\right)$, la correlación que siguió por magnitud fue entre la dimensión conductual y la cognitiva $\left(r_{S}=0.63, p<.01\right)$, y la correlación menor se dio entre las dimensiones conductual y emocional $\left(r_{S}=0.53, p<.01\right)$. 
Respecto a evidencia de validez, se encontraron correlaciones positivas y significativas entre el promedio escolar (rendimiento escolar) reportado por las personas participantes y las tres dimensiones de involucramiento escolar. La correlación más alta se obtuvo con la dimensión conductual $r_{s}=0.46, p<.01$, la segunda en magnitud fue la dimensión cognitiva $r_{S}$ $=0.23, p<.01$, y la tercera fue la dimensión emocional $r_{S}=0.18, p<.01$.

Figura 1: Modelo factorial confirmatorio de la Escala de Involucramiento Escolar para Adolescentes EIE-A (México, 2018-2019)

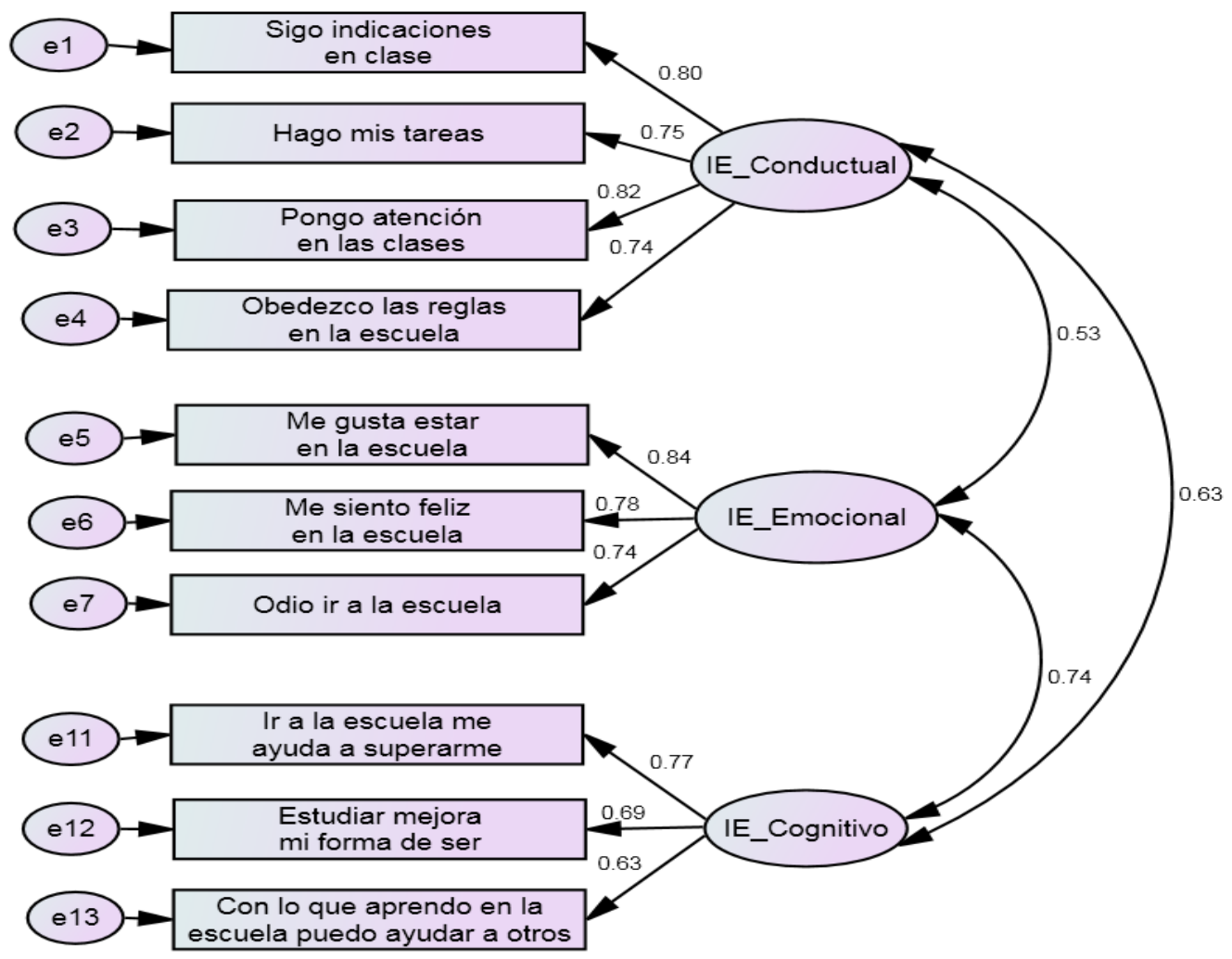

Nota: Dimensiones de Involucramiento Escolar (IE): IE_Conductual = involucramiento escolar conductual, IE_Emocional = involucramiento escolar emocional, IE_Cognitivo= involucramiento escolar cognitivo. $\leftrightarrow$ Correlación entre factores; $\rightarrow$ cargas factoriales estandarizadas.

Fuente: Elaboración propia

Christenson et al. (2012), Fredricks et al. (2004), Fredricks y McColskey (2012), Hernández (2015), Janosz (2012) y Owen et al. (2016) señalan que el involucramiento escolar es un constructo multidimensional. Esto coincide con los resultados obtenidos en esta investigación, ya que en la muestra se confirmó el modelo de tres factores de involucramiento escolar (dimensiones: conductual, emocional y cognitiva). 
Respecto a los instrumentos que miden el involucramiento escolar, algunos coinciden en el número de dimensiones, pero no con la tipología de estas. Por ejemplo la EIE-A, la SES-4DS (Veiga, 2016), el SSEM (Hazel et al., 2013) y el cuestionario de compromiso escolar (Lara et al., 2018) evalúan tres dimensiones del involucramiento escolar, pero el SSEM (Hazel et al., 2013) mide las dimensiones: pertenencia, aspiraciones y productividad; mientras que la EIE-A, la SES-4DS (Veiga, 2016) y el cuestionario de compromiso escolar (Lara et al., 2018) miden las dimensiones: conductual, emocional y cognitiva.

Un aspecto a destacar respecto a las dimensiones del involucramiento escolar es que usar igual denominación de dimensiones no garantiza que los instrumentos midan los mismos aspectos, incluso, se puede encontrar que las escalas evalúan el mismo factor aunque lo nombren de forma distinta. Por ejemplo, cinco de las escalas mencionadas en este estudio evalúan la dimensión cognitiva, no obstante, la SES-4DS (Veiga, 2016) y el cuestionario de compromiso escolar (Lara et al., 2018) miden esfuerzo para comprender ideas y dominar habilidades; el SEI (Appleton et al., 2006) mide aspiraciones futuras y motivación extrínseca y la MES (Martin, 2009) mide cognición adaptativa e impedimento cognitivo. Por otro lado, la EIE-A, en la dimensión cognitiva, mide creencias del alumnado hacia su actividad escolar, y el SSEM (Hazel et al., 2013) en la dimensión aspiraciones, tiene algunos reactivos relacionados con creencias (p.e. tener éxito en la escuela me ayudará en el futuro), lo mismo que el SEI (p.e. mi educación creará muchas oportunidades futuras para mí) (Appleton et al., 2006) en la dimensión adaptación cognitiva, aunque la finalidad de estos dos últimos instrumentos no es la medición de creencias.

En la dimensión conductual, la EIE-A evalúa acciones del alumnado hacia su actividad escolar. Los reactivos que utiliza son semejantes a varios de los que se presentan en el EvsD (Skinner et al., 2009) y en la dimensión de productividad del SSEM (Hazel et al., 2013), por ejemplo, poner atención en clases. En contraste, en el cuestionario de compromiso escolar (Lara et al., 2018) y la SES-4DS (Veiga, 2016), los reactivos de la dimensión conductual hacen referencia a falta de compromiso (p.e. me ausento de clases sin una razón justificada) o mala conducta (p.e. soy grosero con los maestros) por parte del alumnado. En el caso de la EIE-A, los reactivos que se redactaron en sentido negativo se eliminaron en los estudios previos a la versión final de la escala, por no cumplir con los criterios estadísticos mencionados en el apartado de técnicas de recolección de datos.

La EIE-A y el cuestionario de compromiso escolar (Lara et al., 2018) y el EvsD (Skinner et al., 2009) evalúan con reactivos similares la dimensión emocional, por ejemplo: me siento 
feliz en la escuela, me siento bien en este colegio, cuando estoy en clase me siento bien. La EIE-A tiene solo un reactivo negativo (odio ir a la escuela), mientras que otras escalas tienen dimensiones que evalúan varias emociones negativas, como la desafección emocional del EvsD (Skinner et al., 2009) y la dimensión del impedimento cognitivo de la MES (Martin, 2009).

En la dimensión emocional, la EIE-A evalúa emociones del estudiantado hacia su actividad escolar, mientras que otros instrumentos como la SES-4DS (Veiga, 2016) y el cuestionario de compromiso escolar (Lara et al., 2018) evalúan esta dimensión con reactivos positivos y negativos relacionados con pertenencia (p.e. mi escuela es un lugar donde me siento integrado, mi escuela es un lugar donde me siento excluido).

Por último, cabe mencionar que, a pesar de que los reactivos de las dimensiones conductual y emocional de la EIE-A se redactaron con base en la definición propuesta por Fredricks et al. (2004), en los estudios piloto se eliminaron los reactivos acerca de actividades extracurriculares y emociones hacia el personal docente y el estudiantado, los cuales forman parte de la definición de estas dimensiones. En la versión final de la escala solo se conservaron los reactivos referentes a actividades académicas y sentimientos hacia la escuela, no obstante, se requiere realizar más investigación para confirmar si estos aspectos son irrelevantes en el involucramiento escolar de las personas en la adolescencia temprana o si los reactivos se deben redactar de forma diferente para representar estos elementos de las dimensiones.

\section{Conclusiones}

En esta investigación se confirmó un modelo de tres dimensiones de involucramiento escolar con una muestra de personas en adolescencia temprana. La EIE-A tiene coeficientes de consistencia interna e índices de ajuste adecuados, por lo que se puede afirmar que es un instrumento útil para evaluar el involucramiento en el estudiantado de secundaria.

La EIE-A representa una contribución a la medición del involucramiento escolar, pues es una escala breve y su consistencia interna e índices de ajuste son similares o mayores a los obtenidos por instrumentos desarrollados en lengua inglesa, como el SEI (Appleton et al., 2006), el SSEM (Hazel et al., 2013), la MES (Martin, 2009) y el EvsD (Skinner et al., 2009). Asímismo, es similar a instrumentos diseñados en países de América Latina, como el cuestionario de compromiso escolar (Lara et al., 2018) o la versión en portugués y español de la SES-4DS (Veiga, 2016; Tomas et al., 2016). 
Un aporte que hace la EIE-A es proporcionar evidencia empírica de la pertinencia de medir la dimensión cognitiva del estudiantado como creencias hacia su actividad escolar, ya que se encontraron correlaciones positivas y significativas con las dimensiones conductual y emocional, y con el rendimiento académico.

Se sugiere que en futuras investigaciones los resultados obtenidos de los instrumentos se analicen desde métodos cualitativos, además de los estadísticos (Martin, 2009), que se consideren aspectos demográficos en el análisis de resultados (Appleton et al., 2006), que se realicen estudios longitudinales del involucramiento escolar (Samuelsen, 2012) y se observe si la estructura factorial de los instrumentos se mantiene o cambia en muestras de estudiantado de contextos diferentes (Lam et al., 2014; Samuelsen, 2012; Yazzie-Mintz y McCormick, 2012).

Por último, se considera necesario continuar la investigación del involucramiento escolar para mejorar los instrumentos que miden este constructo en la adolescencia temprana. Contar con mejores instrumentos permitirá obtener resultados que orienten el desarrollo de estrategias para fomentar el involucramiento y lograr sus beneficios, como alto rendimiento escolar, bienestar (Darr, 2012; González-Brignardello y Sánchez-ElviraPaniagua, 2013), evitar burnout escolar (Parra y Pérez, 2010) y conductas de riesgo (Shoshani, Nakash, Zubida y Harper, 2016; Wang y Fredricks, 2014).

\section{Agradecimientos}

La becaria María Enriqueta Sánchez Hernández, con número 211502 y Currículum Vitae Único (CVU) 234993, agradece al Consejo Nacional de Ciencia y Tecnología (CONACYT) el apoyo brindado.

\section{Referencias}

Appleton, James, Christenson, Sandra, Kim, Dongjin and Reschly, Amy. (2006). Measuring cognitive and psychological engagement: validation of the student engagement instrument. Journal of School Psychology, 44, 427-445.

Appleton, James, Christenson, Sandra y Furlong, Michael. (2008). Student engagement with school: Critical conceptual and methodological issues of the construct. Psychology in the Schools, 45(5), 369-386.

Benson, Peter. (2007). Developmental assets: an overview of theory, research and practice. En Rainer Silbereisen y Richard Lerner (Eds.), Approaches to positive youth development (pp. 1-58). Gran Bretaña: TJ International Ltd, Padstow, Cornwall. 
Buelga, Sofía; Cava, María Jesús y Musitu, Gonzalo. (2012). Reputación social, ajuste psicosocial y victimización entre adolescentes en el contexto escolar. Anales de Psicología, 28(1), 180-87.

Christenson, Sandra; Reschly, Amy and Wylie, Cathy. (2012). Handbook of research on student engagement. London: Springer New York Dordrecht Heidelberg.

Darr, Charles. (2012). Measuring student engagement: the development of a scale for formative use. En Sandra Christenson, Amy. Reschly, \& Cathy Wylie (Eds.) Handbook of research on student engagement (pp.707-723). London Springer New York Dordrecht Heidelberg.

Daura, Florencia Teresita. (2015). El estudio del compromiso académico, panorama general sobre su abordaje. Diálogos Pedagógicos, 13(25), 54-75.

Dolzan, Martina; Sartori, Riccardo; Charkhabi, Morteza y De Paola, Francesco. (2015). The effect of school engagement on health risk behaviours among high school students: testing the mediating role of self-eficacy. Procedia - Social and Behavioral Sciences, 205, 608-613. Recuperado de https://cyberleninka.org/article/n/569270

Embretson, Susan y Gorin, Joanna. (2001). Improving construct validity with cognitive psychology principles. Journal of Educational Measurement, 38(4), 343-368.

Estell, David y Perdue, Neil. (2013). Social support and behavioral and affective school engagement: The effects of peers, parents, and teachers. Psychology in the Schools, 50(4), 325-339.

Fernández-Zabala, Arantza; Goñi, Eider; Camino, Igor y Zulaika, Luis Mari. (2015). Family and school context in school engagement. European Journal of Education and Psychology, 9(2), 47-55.

Finn, Jeremy. (1993). School engagement and students at risk. Washington, DC: National Center for Education Statistics. Recuperado de http://citeseerx.ist.psu.edu/viewdoc/download?doi=10.1.1.545.5418yrep=rep1 ytype=pdf

Fredricks, Jennifer; Blumenfeld, Phyllis y Paris, Alison. (2004). School engagement: Potential of the concept, state of the evidence. Review of Educational Research, 74(1), 59-109.

Fredricks, Jennifer y McColskey, Wendy. (2012). The measurement of student engagement: a comparative analysis of various methods and student self-report instruments. En Sandra Christenson, Amy Reschly, \& Cathy Wylie (Eds.), Handbook of research on student engagement, (pp 763-782). London: Springer New York Dordrecht Heidelberg.

Glanville, Jennifer y Wildhagen, Tina. (2007). The measurement of school engagement assessing dimensionality and measurement invariance across race and ethnicity. Educational and Psychological Measurement, 67(6), 1019-1041. doi: org/10.1177/0013164406299126 
González-Brignardello, Marcela Paz y Sánchez-Elvira-Paniagua, Ángeles. (2013). ¿Puede amortiguar el engagement los efectos nocivos de la procrastinación académica? Acción Psicológica, 10(1), 117-134.

Gutiérrez, Melchor: Tomás, José-Manuel; Romero, Isabel y Barrica, José-Marcos. (2017). Perceived social support, school engagement and satisfaction with school. Revista de Psicodidáctica, 22(2), 111-117.

Hazel, Cynthia; Vazirabadi, Emma y Gallagher, John. (2013). Measuring aspirations, belonging, and productivity in secondary Students: validation of the student school engagement measure. Psychology in the Schools, 50(7), 689-704. doi: 10.1002/pits.21703

Hernández, Berkys. (2015). Involucramiento de escolares de enseñanza media dominicanos: antecedentes y consecuentes (Tesis doctoral). Universidad de Valencia, Valencia, España. Recuperada de https://docplayer.es/45088420-Involucramiento-de-escolaresde-ensenanza-media-dominicanos-antecedentes-y-consecuentes.html

Hu, Li-tze y Bentler, Peter. (1999). Cutoff criteria for fit indices in covariance structure analysis: Conventional criteria versus new alternatives. Structural Equation Modeling: $A$ Multidisciplinary Journal, 6(1), 1-55.

Janosz, Michel. (2012). Part IV commentary: outcomes of engagement and engagement as an outcome: some consensus, divergences, and unanswered questions. En Sandra Christenson, Amy Reschly and Cathy Wylie (Eds.), Handbook of research on student engagement (pp. 695-703). London: Springer New York Dordrecht Heidelberg.

Kim, Ha y Suárez-Orozco, Carola. (2015). The language of learning: the academic engagement of newcomer immigrant youth. Journal of Research on Adolescence, 25(2), 229-245.

Lam, Shui; Jimerson, Shane; Wong, Bernard; Kikas, Eve, Shin, Hyeonsook, Veiga, Feliciano, Hatzichristou, Chryse, Polychroni, Fotini, Cefai, Carmel, Negovan, Valeria, Stanculescu, Elena, Yang, Hongfei, Liu, Yi, Basnett, Julie, Duck, Robert, Farrell, Peter, Nelson, Brett y Zollneritsch, Josef. (2014). Understanding and measuring student engagement in school: The results of an international study from 12 countries. School Psychology Quarterly, 29(2), 213-232.

Lara, Laura; Saracostti, Mahia; Navarro, Juan-José; De-Toro, Ximena; Miranda-Zapata, Edgardo; Trigger, Jennifer Marie y Fuster, Jaime. (2018). Compromiso escolar: Desarrollo y validación de un instrumento. Revista Mexicana de Psicología, 35(1), 5262.

Libbey, Heather. (2004). Measuring students' relationship to school: Attachment, bonding, connectedness, and engagement. Journal of School Health, 74(7), 274-283.

Martin, Andrew. (2009). Motivation and engagement across the academic life span: a developmental construct validity study of elementary school, high school, and university/college students. Educational and Psychological Measurement, 69(5), 794824. 
Owen, Katherine; Parker, Philip; Van-Zandez, Brooke; MacMillan, Freya; Astell-Burt, Thomas and Lonsdale, Chris. (2016). Physical activity and school engagement in youth: a systematic review and meta-analysis. Educational Psychologist, 51(2), 129-145.

Pan, Jingtong y Zaff, Jonathan. (2017). The measurement structure of school engagement among youth in China: an exploratory structural equation modeling study. Journal of Psychoeducational Assessment, 37(3), 280-292.

Parra, Paula. (2010). Relación entre el nivel de engagement y el rendimiento académico teórico/práctico. Revista de Educación en Ciencias de la Salud, 7(1), 57-63.

Parra, Paula y Pérez, Cristhian. (2010). Propiedades psicométricas de la escala de compromiso académico, UWES-S (versión abreviada), en estudiantes de psicología. Revista de Educación en Ciencias de la Salud, 7(2), 128-133.

Peña, Gustavo; Cañoto, Yolanda y Angelucci, Luisa. (2017). Involucramiento académico: una escala. Revista Páginas de Educación, 10(1), 114-136. doi.org/10.22235/pe.v10i1.1361

Peña, Juan Carlos; Soto, Valeria Edith y Calderón, Uranía Alixon. (2016). La influencia de la familia en la deserción escolar: estudio de caso en estudiantes de secundaria de dos instituciones de las comunas de Padre las Casas y Villarrica, Región de la Araucanía, Chile. Revista Mexicana de Investigación Educativa, 21(70), 881-899.

Ramos-Díaz, Estibaliz; Rodríguez-Fernández, Arantzazu; Ros, Iker y Antonio-Aguirre, Iratxe. (2016). Implicación escolar y autoconcepto multidimensional en una muestra de estudiantes españoles de secundaria. Revista Complutense de Educación, 28(4), 1103-1118.

Real Academia de la Lengua. (2019). Diccionario de la lengua española. Definición de compromiso. Recuperado de https://dle.rae.es/?id=A41ilou

Rodríguez-Fernández, Arantzazu; Ramos-Díaz, Estibaliz; Fernández-Zabala, Arantza; Goni, Eider; Esnaola, Igor y Goni, Alfredo. (2016). Contextual and psychological variables in a descriptive model of subjective well-being and school engagement. International Journal of Clinical and Health Psychology, 16(2), 166-174.

Samuelsen, Karen. (2012). Part V Commentary: Possible new directions in the measurement of student engagement. En Sandra Christenson, Amy Reschly, \& Cathy Wylie (Eds.), (Eds.), Handbook of research on student engagement (pp. 805-811). London: Springer New York Dordrecht Heidelberg.

Schaufeli, Wilmar; Salanova, Marisa; González-Romá, Vicente y Bakker, Arnold. (2002). The measurement of engagement and burnout: A two sample confirmatory factor analytic approach. Journal of Happiness Studies, 3, 71-92.

Shoshani, Anat, Nakash, Ora, Zubida, Hani, \& Harper, Robin. (2016). School engagement, acculturation, and mental health among migrant adolescents in Israel. School Psychology Quarterly, 31(2), 181-197. 
Skinner, Ellen; Kindermann, Thomas y Furrer, Carrie. (2009). A motivational perspective on engagement and disaffection: conceptualization and assessment of children's behavioral and emotional participation in academic activities in the classroom. Educational and Psychological Measurement, 69(3), 493-525.

Skinner, Ellen; Furrer, Carrie; Marchand, Gwen y Kinderman, Thomas. (2008). Engagement and disaffection in the classroom: Part of a larger motivational dynamic? Journal of Educational Psychology, 100(4), 765-781.

Tarabini, Aina. (2015). La meritocracia en la mente del profesorado: un análisis de los discursos docentes en relación al éxito, fracaso y abandono escolar. Revista de la Asociación de Sociología de la Educación, 8(3), 349-360.

Tomás, José; Gutiérrez, Melchor; Sancho, Patricia; Chireac, Silvia y Romero, Isabel. (2016). El compromiso escolar (school engagement) de los adolescentes: medida de sus dimensiones. Enseñanza \& Teaching, 34(1), 119-135.

Upadyaya, Katja y Salmela-Aro, Katariina. (2013). Development of school engagement in association with academic success and well-being in varying social contexts: A review of empirical research. European Psychologist, 18(2), 136-147.

Veiga, Feliciano. (2016). Assessing student engagement in school: Development and validation of a four-dimensional scale. Procedia - Social and Behavioral Sciences, 217, 813-819.

Veiga, Feliciano y Robu, Viorel. (2014). Measuring student engagement with school across cultures: psychometric findings from Portugal and Romania. Romanian Journal of School Psychology, 7(14), 10-20.

Wang, Ming-Te y Eccles, Jacquelynne. (2012). Adolescent behavioral, emotional, and cognitive engagement trajectories in school and their differential relations to educational success. Journal of Research on Adolescence, 22(1), 31-39.

Wang, Ming-Te y Holcombe, Rebecca. (2010). Adolescents' perceptions of school environment, engagement, and academic achievement in middle school. American Educational Research Journal, 47(3), 633-662. doi:10.3102/0002831209361209

Wang, Ming-Te y Fredricks, Jennifer. (2014). The reciprocal links between school engagement, youth problem behaviors, and school dropout during adolescence. Child Development, 85(2), 722-737. doi:10.1111/cdev.12138

Yazzie-Mintz, Ethan y McCormick, Kim. (2012). Finding the humanity in the data: understanding, measuring, and strengthening student engagement. En Sandra Christenson, Amy Reschly, \& Cathy Wylie (Eds.), Handbook of research on student engagement (pp.743-761). London: Springer New York Dordrecht Heidelberg. 
Revista indizada en

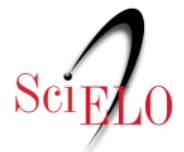

redalyc satindex

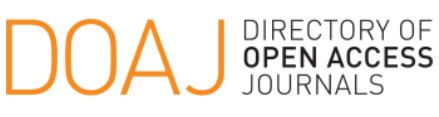

Distribuida en las bases de datos:

- Dialnet

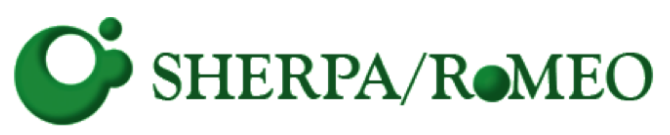

REDIB

Red Iberoamericana

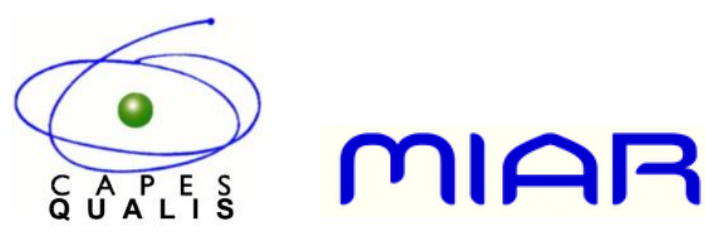

